\title{
Cancer occurrence in Danish diabetic patients: duration and insulin effects
}

\author{
B. Carstensen • D. R. Witte • S. Friis
}

Received: 5 April 2011 /Accepted: 31 October 2011 /Published online: 27 November 2011

(C) Springer-Verlag 2012

\begin{abstract}
Aims/hypothesis Cancer is more frequent among diabetes patients, but it is unknown how this excess varies with duration of diabetes and insulin use. The aim of this study was to analyse disease data to examine this issue further. Methods We linked the Danish National Diabetes Register and Cancer Registry and performed a cohort analysis of the entire Danish population by diabetes status, duration of diabetes and insulin use, comparing cancer incidence rates in diabetic patients with the non-diabetic population for the 15 year period 1995-2009, using Poisson regression with natural splines to describe the variation by duration.

Results We found 20,032 cancer cases among patients not using insulin and 2,794 cancer cases among diabetic patients using insulin. The cancer incidence rate ratio among non-insulin users relative to the non-diabetic
\end{abstract}

Electronic supplementary material The online version of this article (doi:10.1007/s00125-011-2381-4) contains peer-reviewed but unedited supplementary material, which is available to authorised users.

B. Carstensen $(\bowtie)$

Steno Diabetes Center,

Niels Steensens Vej 2,

2829 Gentofte, Denmark

e-mail: bxc@steno.dk

B. Carstensen - D. R. Witte

Department of Biostatistics, University of Copenhagen,

Copenhagen, Denmark

S. Friis

Institute of Cancer Epidemiology, Danish Cancer Society, Copenhagen, Denmark

\section{S. Friis}

Institute of Public Health, University of Copenhagen,

Copenhagen, Denmark population decreased from over 2 at diagnosis to 1.15 after 2 years of diabetes duration. The cancer incidence rate ratio was higher among patients using insulin, decreasing from 5 at the start of insulin treatment to about 1.25 after 5 years of insulin use. Among non-insulin users, cancers of the stomach, colorectum, liver, pancreas, lung, corpus uteri, kidney and brain, and lymphomas were elevated. Among insulin users the rate ratio of prostate cancer was decreasing by duration whereas we found higher risk of cancer of the stomach, lung, liver, pancreas and kidney. Breast cancer incidence rates were not affected by either diabetes or insulin use.

Conclusions The observed duration effects suggest that both increased surveillance for cancer in the first years after diagnosis of diabetes, and reverse causation, where undiagnosed cancers increase the likelihood of diabetes diagnosis, play a role. For longer durations, a combination of common causes for diabetes and cancer, as well as the effects of diabetes and insulin exposure per se, may play a role in the association between diabetes and some cancers.

Keywords Cancer incidence - Cancer rate ratio . Cumulative risk of cancer - Diabetes duration - Insulin duration · Insulin treatment · Mortality · Register study . Prescriptions

$\begin{array}{ll}\text { Abbreviations } \\ \text { APC } & \text { Age-period-cohort model } \\ \text { CPR } & \text { Central Person Register } \\ \text { DCR } & \text { Danish Cancer Registry } \\ \text { DM } & \text { 'Diabetes, no insulin' category in analysis } \\ \text { DM+Ins } & \text { 'Diabetes and insulin' category in analysis } \\ \text { NDR } & \text { (Danish) National Diabetes Register } \\ \text { No DM } & \text { 'No diabetes' category in analysis }\end{array}$




\section{Introduction}

Diabetic patients carry a higher risk of cancer than the general population and this excess risk differs by cancer site [1-3]. The explanation has remained elusive, as the low incidence of any given cancer means that most studies in the field have been observational by necessity and therefore open to confounding that cannot be remedied. A number of studies have addressed the question of the relationship between drug use and cancer occurrence among diabetic patients, particularly with respect to the effect of oral glucose-lowering drugs [4]. A study of the cancer incidence in insulin users found a twofold increased risk of colorectal cancer among insulin users. Several studies have reported an inverse association between diabetes and subsequent prostate cancer $[5,6]$. However, the nature of the link between the risk of cancer and both the duration of diabetes and insulin use is not well described, let alone understood.

The aim of this study was to relate the occurrence of cancer among diabetic patients to the non-diabetic part of the population and, in particular, to describe how this relation varies by duration of diabetes and insulin treatment.

\section{Methods}

\section{Register data}

The study covered the entire Danish population in the 15 year period from 1 January 1995 to 31 December 2009, based on register linkage. We used registers to retrieve the date of birth, date of cancer diagnosis, date of diabetes diagnosis, date of first insulin use and date of death for all persons in the population.

Central Person Register Since 1 April 1968, all individuals with permanent residence in Denmark have been given a unique identification number (Central Person Register [CPR] number) [7], which is used for all administrative purposes in Denmark and, in particular, all health events recorded in registers are identified by the CPR number, and so are uniquely linkable.

The National Diabetes Register The National Diabetes Register (NDR) was established in 2006, and currently contains records of all prevalent cases of diabetes as of 1 January 1995 and all incident cases of diabetes up to 31 December 2009 [8, 9]. The start of insulin treatment is defined as the date of the second insulin purchase in the prescription database.

The recorded date of inclusion in the NDR is only reliable after 1 January 1995. Therefore, duration of diabetes and insulin use can only be determined reliably for individuals included in the register after that date.

The Danish Cancer Registry The Danish Cancer Registry (DCR) was established in 1943 [10, 11], and contains details of all tumours diagnosed in Denmark between 1 January 1943 and 31 December 2009, a total of 1.25 million cancers among 1.15 million individuals. All patients alive after 1 April 1968, when the CPR was established, are identified by their CPR number.

Cancers in this study were defined as the first primary cancer only, in order to avoid diagnostic artefacts and possible effects from treatment of the first cancer. Moreover, non-melanoma skin cancer was not counted as a cancer in this study.

\section{Linkage and tabulation}

Individuals were followed from 1 January 1995 to 31 December 2009, or to death or diagnosis of any cancer, whichever occurred first. All prevalent cases of cancer and diabetes as of 1 January 1995 were excluded. Follow-up was classified in the categories 'No DM' (no diabetes), 'DM' (diabetes, no insulin) and 'DM+Ins' (diabetes and insulin treatment). Person-years and the number of incident cancers were enumerated for 25 different groups of cancer diagnoses, as were deaths prior to cancer diagnosis (Table 1). Cancers and followup during the first month after diabetes diagnosis were excluded to avoid uncertainty in the sequence of diabetes and cancer diagnoses (details in the electronic supplementary material [ESM] [Fig. 1, and Chapter 1.2 'Accuracy of dates']).

The person-years in the Danish population were based on figures from Statistics Denmark and calculated as indicated in [12-14]. The person-years in the 'no DM' category were computed as the person-years in the total Danish population minus the person-years lived by persons diagnosed with cancer or diabetes.

The follow-up (person-years and cancer cases) was classified by diabetes and treatment category, sex, age and calendar time at follow-up, and date of birth in 1 year classes (Lexis triangles [12]). Follow-up in the two diabetic categories was further subdivided by time since inclusion in the register (diabetes duration), and by time since the second insulin purchase (insulin duration), in intervals of length 3, 6, 12 and 24 months, as illustrated in Fig. 2.

All data manipulation and tabulation was performed in the SAS system, using the macro\%Lexis, [15]. This is documented in detail in the links provided in the ESM. 


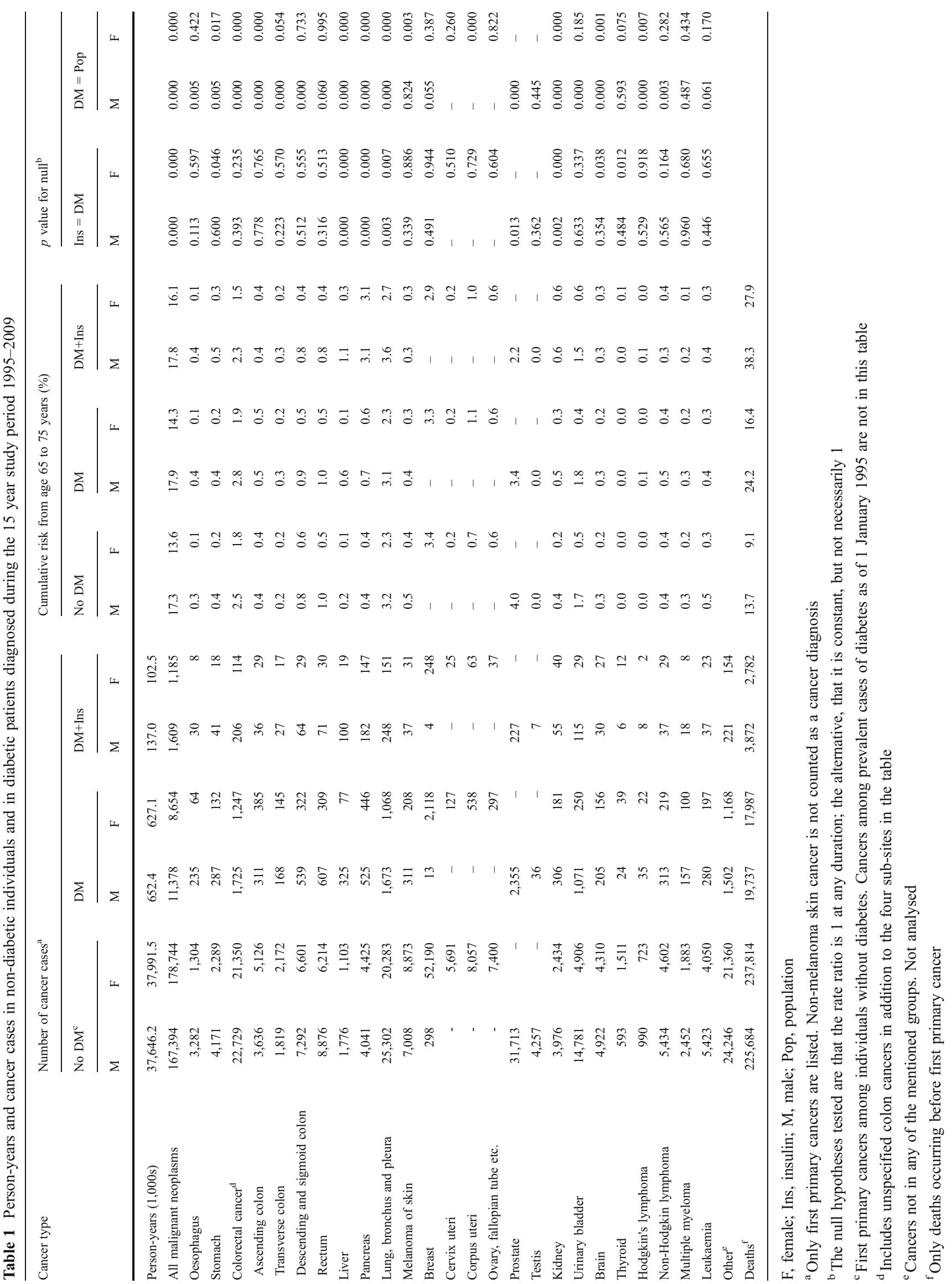


Fig. 1 Estimated rate ratios (with $95 \%$ CIs) of cancer occurrence for diabetes patients not using insulin (dark red, women; dark blue, men) and diabetes patients using insulin (light red, women; light blue, men) vs non-diabetic patients for different cancers. The rate ratios for cancer of the liver, pancreas and testis in insulintreated patients are outside the figure; they are: liver, men 8.1 $(6.6,9.9)$, women: $4.0(2.6,6.3)$ pancreas, men $6.9(6.0,7.9)$, women $7.2(6.2,8.4)$; testis $0.50(0.24,1.05)$

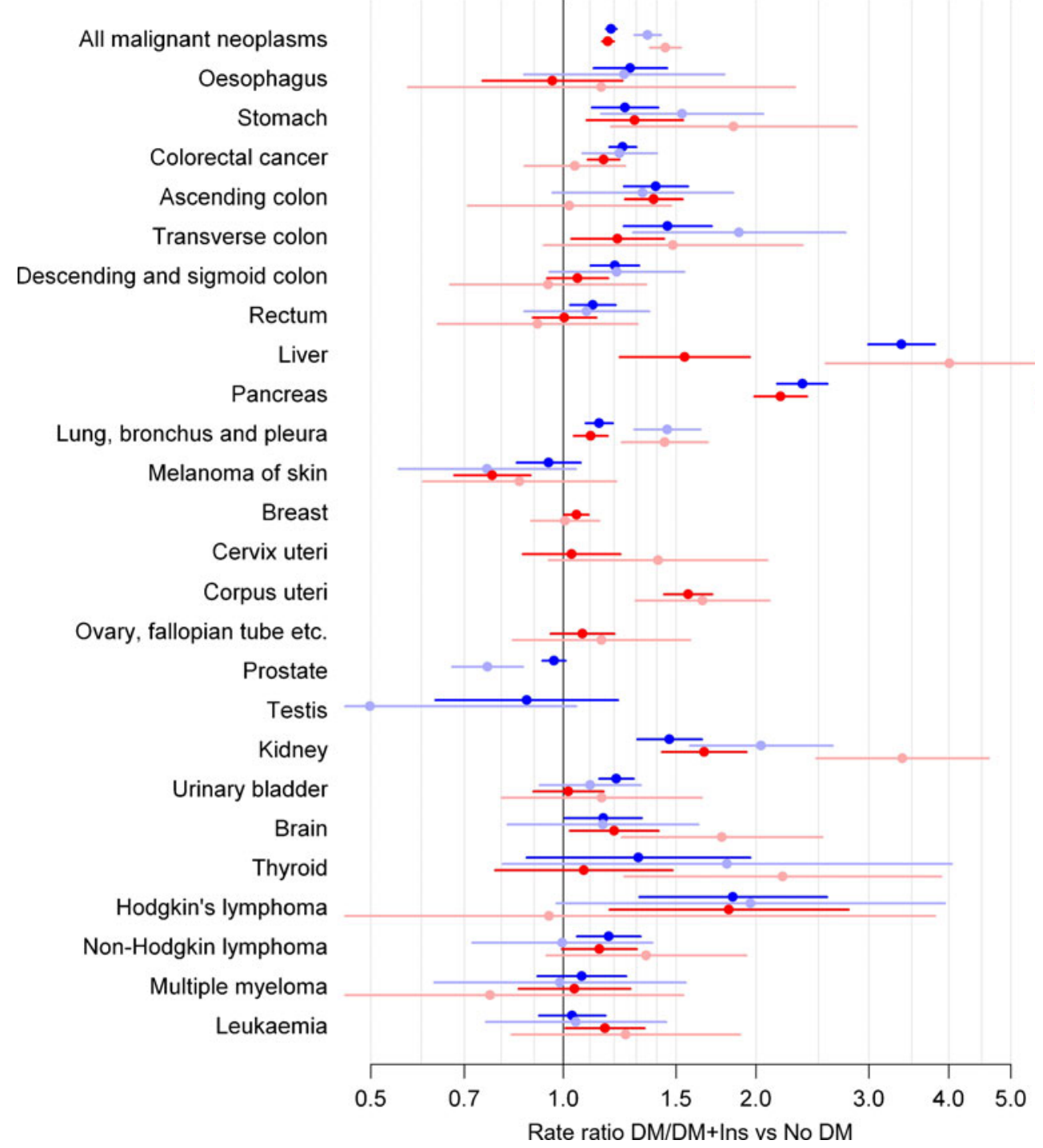

and whether diabetic patients had the same risk as the nondiabetic population.

In an extended model, the rate ratio relative to the nondiabetic population was taken as a function of diabetes duration and (for insulin users), insulin duration, modelled by restricted cubic (natural) splines with four and three parameters, respectively, (for the outcome 'all cancer', five and four parameters, respectively) [16]. We chose the first knot to be 0 and the remaining knots so that the number of incident cancers between each pair of knots, and after the last knot, was the same.

Interaction between insulin and diabetes duration was tested by including two extra terms: diabetes duration at insulin start, and the product of diabetes and insulin duration.

The rate ratio is shown graphically by duration of diabetes for diabetic patients not taking insulin, as well as for diabetic patients initiating insulin at 0,3 and 5 years after diagnosis. 


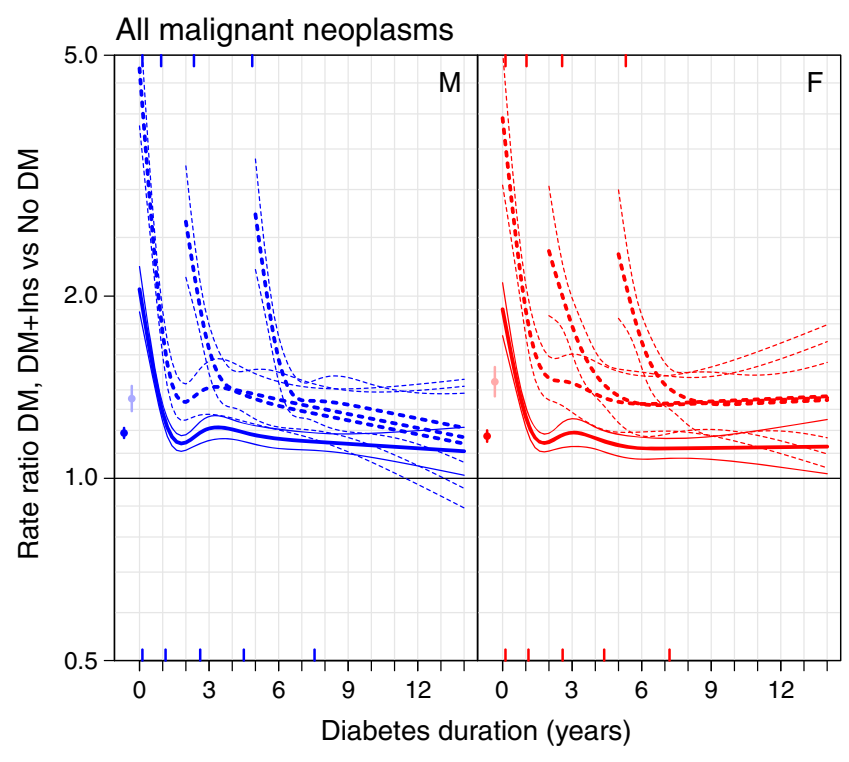

Fig. 2 Cancer incidence rate ratio vs the non-diabetic population for all malignant neoplasms. Spline models with five (diabetes duration) and four (insulin duration) parameters. Blue, men; red, women; solid lines, diabetic patients not using insulin; dashed lines, patients using insulin, starting insulin after 0,2 or 5 years of diabetes duration, respectively, moving from left to right; thin lines indicate $95 \%$ CIs. The bars on the left are estimates from the model ignoring duration effects, and the blue and red ticks at the top and bottom inside indicate the location of the knots for the spline functions; the number of cancers between each pair of knots and to the right of the last are the same. F, female; $\mathrm{M}$, male

Cumulative risk To illustrate how the estimated rates and rate ratios translate into risk of cancer, we computed the 10 year cumulative risk of each particular type of cancer from age 65, 70 or 75 for a person in each of the categories 'No DM', 'DM' and 'DM+Ins'. The follow-up was assumed to cover the period 1 January 1998 to 31 December 2009. We accounted for death as a competing risk to cancer [17-19] by using mortality rates estimated from APC-models for death, fitted separately for the categories 'No DM', 'DM' and 'DM+Ins'.

Terminology We used the term 'significant' for estimates that were more than twice as large as the estimated standard error, even though the formal statistical machinery relies heavily on the correctness of the models used. Likewise, the $95 \%$ CIs used were based on the correctness of the models, and the validity of the normal approximation to the distribution of the parameter estimates.

All statistical analyses and graphical reporting of results were carried out in R [20]. Results are documented in more detail in the ESM figures.

\section{Results}

Figure 1 shows the overall rate ratios of cancer relative to the non-diabetic population for individuals with diabetes, subdivided by insulin use. The rate ratios among diabetes patients using insulin and those not on insulin followed largely the same pattern with respect to site of cancer, with a general tendency toward higher rate ratios among those using insulin. For all cancers combined, the rate ratio was 1.2 for patients not on insulin and 1.4 for patients on insulin, with very slight differences between the sexes.

In the non-insulin group, there were significantly elevated rate ratios for cancer of the stomach, colorectum, liver, pancreas, lung, corpus uteri, kidney and brain, and for lymphomas, whereas elevated rates among insulin users were seen for the stomach, liver, pancreas, lung, corpus uteri and kidney. The rate ratios in the insulin group were generally higher, but fewer were significant.

The rate ratio for liver cancer was 3.4 among men and 1.6 among women not using insulin, but more than twice as high among those using insulin (8.1 and 4.0, respectively). For pancreatic cancer, the rate ratio was 2.4 (male) and 2.2 (female) for patients not on insulin, and three times as high (6.9 for men and 7.2 for women) among those on insulin. The rate ratio for prostate cancer among patients using insulin was 0.8 , whereas that for patients not on insulin was 1.0 .

We found a decreasing tendency for the rate ratio with more distant locations along the digestive tract, with the highest rate ratio for stomach cancer and smallest for rectal cancer (see Fig. 1).

Within the group of diabetic patients, we found significantly higher cancer incidence rates among insulin users relative to non-users for all cancers combined, and for cancers of the liver, pancreas, lung and kidney, and smaller rates of prostate cancer, (see Fig. 1 and the figure of rate ratios comparing users and non-users shown in the ESM). No other sites exhibited a consistent pattern in the overall effect of diabetes or insulin treatment on cancer risk.

\section{Duration effects}

Among the 25 sites analysed by sex, we only found a significant interaction between duration of diabetes and insulin for two sites (liver, among men and thyroid, among women). Moreover, the inclusion of the estimated interaction in the reporting of the effects makes virtually no difference in the curves (data not shown). Hence the model that did not include interaction between the durations was used for reporting throughout.

The general shape of the rate ratio curve by duration showed a high risk at the beginning, decreasing during the first year to a fairly moderate level. The shapes of the rate ratio curves were quite similar for insulin users and nonusers (Fig. 2). For all cancers combined, the rate ratio among non-users was highest in the period just after diagnosis with a rate ratio of 2.0 decreasing to 1.15 after 
2 years. For insulin users the rate ratio started at 5, decreasing to 1.3 after about 3 years. Among men, there was a slight tendency for rate ratios to decline for longer insulin duration. The rate ratios were statistically significant over the entire study period, with the exception of male insulin users, where the effect was not significant after 11 years. For all cancers combined, the rate ratio between insulin users and non-users was about 2.5 at initiation of insulin, about 1.2 after 4 years of insulin use, and largely constant for women, but slightly declining for men (see Fig. 3). These rate ratios were significant for the first $7-$ 9 years of insulin duration.

The effects of duration of diabetes and insulin therapy are shown in Figs 4 and 5 for the following cancer sites: stomach, colorectum, lung, prostate, breast, ovary, corpus uteri, kidney, urinary bladder, liver, pancreas and skin (melanoma). These are the sites with the highest number of cancer cases among diabetic patients; similar figures for all analysed sites are included in the ESM.

Stomach cancer showed a constant rate ratio of about 1.2 for the non-insulin-treated diabetic patients (non-significant when modelled by duration), and an indication of a strong duration effect among insulin-using women only. Colorectal cancer showed a long-term elevated rate ratio of about 1.2 among non-insulin-treated patients, and no indication of a difference between insulin users and non-users. For lung cancer, we did not find a long-term elevated rate ratio among non-insulin users, but among insulin users, the long-term rate ratio was about 1.4 for both sexes.

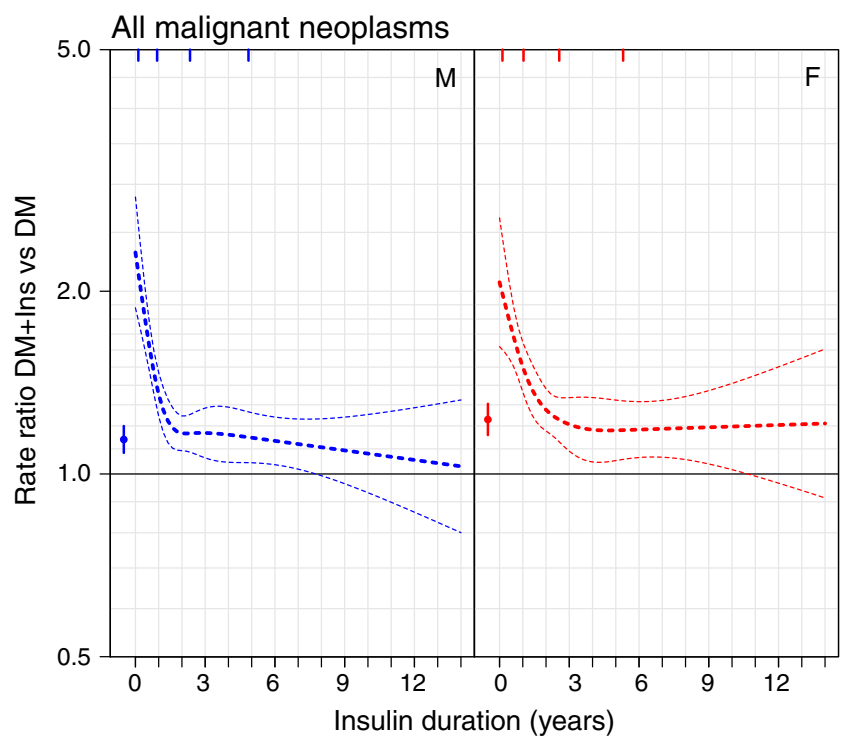

Fig. 3 Cancer incidence rate ratio for insulin users vs non-users for all malignant neoplasms. Spline models with five (diabetes duration) and four (insulin duration) parameters. Blue, men; red, women. Thin lines indicate $95 \%$ CIs. The bars on the left are estimates from the model ignoring duration effects, and the coloured ticks inside indicate the location of the knots for the spline functions. F, female; M, male
For melanoma of the skin, we saw a tendency towards a rate ratio below 1 with increasing duration among women, both for insulin users and non-users, whereas there was no indication of an effect in either direction for men. There was no allocation effect (initially increased rate ratio) for melanoma of the skin in either sex. The smaller incidence rates of prostate cancer among insulin-treated patients were accentuated by longer diabetes duration or insulin treatment. There was no indication of a breast cancer rate ratio different from 1 in any direction, regardless of insulin treatment or duration. Cancer of the corpus uteri showed a rate ratio of around 1.6 at all durations with no indication of any duration effects apart from a small initial allocation effect.

Liver cancer showed duration patterns for users and nonusers of insulin, which were consistent with a largely constant rate ratio. Pancreas cancer showed a dramatic decrease in rate ratio by both diabetes and insulin duration. Kidney cancer showed an initial decrease in rate ratios for both sexes, and for men, we found a long-term constant rate ratio of around 1.3 for non-users and 1.7 for users of insulin (not significantly different), whereas there were higher rate ratios among women, and even an indication of an increasing rate ratio with longer duration of diabetes. The rate ratio between users and non-users of insulin was constant around 2 and significant for the first 8 years of insulin use. No consistent patterns by diabetes duration and insulin duration were seen for cancers of the ovary or urinary bladder.

\section{Cumulative risk}

The 10 year cumulative risks of cancer or dying without cancer are shown in Fig. 6. The differences in cancer incidence rates between the groups were largely dominated by the mortality differences, and the cumulative risk of cancer was approximately the same for the three groups. As a result, the 10 year cumulative risks of cancer essentially only varied by sex (male, $17-18 \%$; female, $14-16 \%$, for 65 year olds). Table 1 presents site-specific cumulative cancer risks for each of the cancer sites from age 65 to 75 years, assuming that follow-up began in 1998 at diabetes diagnosis and start of insulin.

The ESM contains graphs of rate ratios for all sites analysed. A complete account of all analyses reported, including SAS and R-code, can be found at the first author's website: http://BendixCarstensen.com/DMCa/Diabetologia/ Analyses.pdf.

\section{Discussion}

In this study, we compared the cancer occurrence in newly diagnosed Danish diabetic patients with the non-diabetic 
Fig. 4 a-f Rate ratio for selected sub-sites vs the non-diabetic population. Results from spline models with four (diabetes duration) and three (insulin duration) parameters. Blue, men; red, women; solid lines, diabetic patients not using insulin; dashed lines, patients using insulin, starting insulin after 0,2 or 5 years of diabetes duration, respectively, moving from left to right; thin lines indicate $95 \%$ CIs. The bars on the left are estimates from the model ignoring duration effects, and the coloured ticks inside indicate the location of the knots for the spline functions; the number of cancers between each pair of knots and to the right of the last are the same. F, female; $M$, male
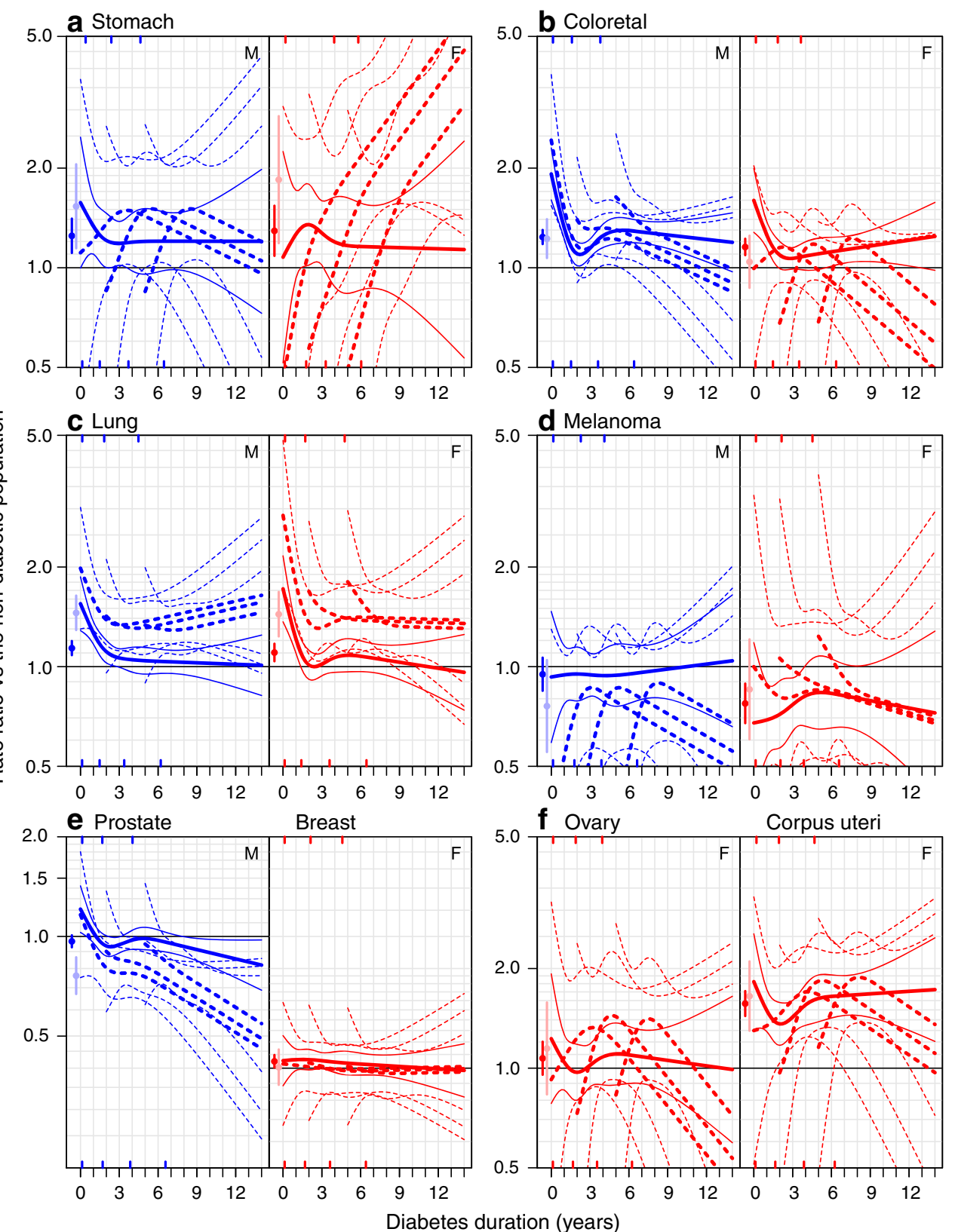

part of the population, and we compared patients using insulin with patients not on insulin. For the first time in the literature, we have simultaneously modelled both inception and duration effects of diabetes and insulin treatment.

We found that the risk of cancer decreased substantially for most sites by duration of diabetes or insulin use. The elevated cancer risk observed just after diabetes diagnosis and the marked decrease during the first 2 years following diagnosis were seen for almost all sites of cancer. This indicates the presence of a detection bias, in the sense that the diagnosis of diabetes leads to increased medical attention, and thus to earlier detection of any present but undiagnosed cancer.
The large number of patients with dates of diagnosis of cancer and diabetes very close to each other (excluded from analysis because of lack of evidence that diabetes preceded cancer; see ESM [Chapter 1.2, 'Accuracy of dates']) also suggests that more or less concomitant clinical manifestation or registration of cancer and diabetes may be quite frequent.

The stable level of the rate ratio seen beyond the initial 2 year period may reflect the combined effect of risk factors common to diabetes and cancer, such as obesity and lifestyle factors, as well as conditions that can precede diabetes, characterised by moderately elevated blood glucose and insulin resistance. Stocks [21] found that there was an effect 
Fig. 5 Rate ratio for selected sub-sites versus the non-diabetic population. Results from spline models with four (diabetes duration) and three (insulin duration) parameters. Blue, men; red, women; solid lines, diabetic patients not using insulin; dashed lines, patients using insulin, starting insulin after 0,2 or 5 years of diabetes duration, respectively, moving from left to right; thin lines indicate $95 \%$

CIs. Cancers of (a) liver,

(b) pancreas, (c) kidney and (d) urinary bladder. The bars on the left are estimates from the model ignoring duration effects, and the coloured ticks inside indicate the location of the knots for the spline functions; the number of cancers between each pair of knots and to the right of the last are the same. F, female; $\mathrm{M}$, male
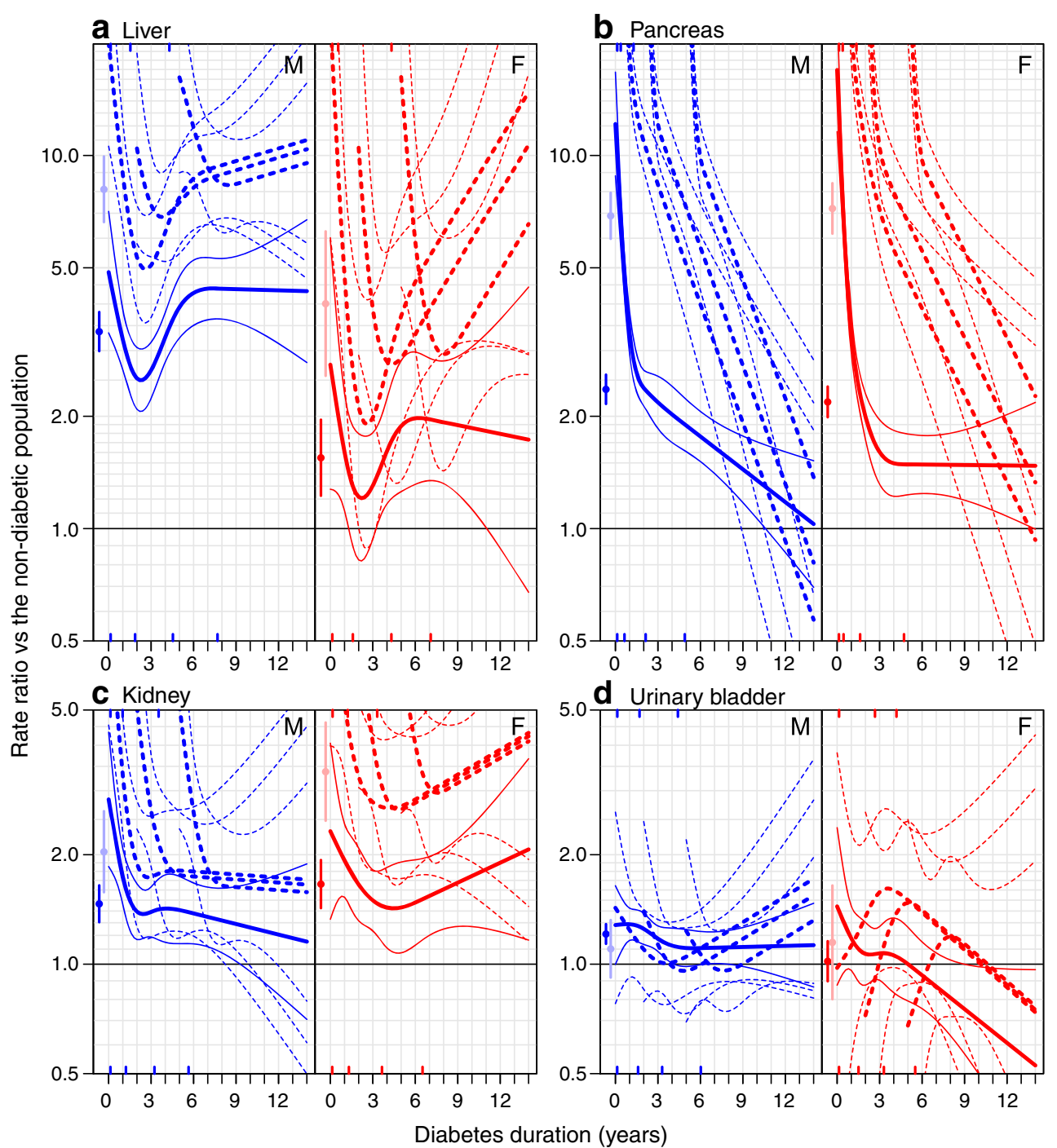

of higher blood glucose levels on the occurrence of cancers, particularly for higher levels of blood glucose with a pattern of elevated rate ratios across cancer sites not very different from the one seen in this study. Moreover, glucoselowering drugs may have an effect on cancer risk, as indicated by the higher cancer rates seen in patients using sulfonylureas relative to those using metformin [4].

Insulin effects

We observed the largest rate ratio between the insulintreated and the non-diabetic populations at the time of initiation of insulin treatment, with a sharp drop in rate ratio during the first year, regardless of the time of insulin initiation relative to diabetes onset (a lack of interaction). This suggests an indication bias: as insulin is generally the third or fourth treatment option, the clinical decision to initiate treatment depends on failure of earlier treatment, or contra-indications to glucose-lowering drugs, such as heart failure or renal or hepatic conditions, which are indicative of general ill health. To the extent that this is linked to undiagnosed (or emerging) cancers, a high rate ratio can be expected in people who have recently started insulin treatment. Moreover, detection bias may also play a role, where people with a recent insulin prescription are checked more thoroughly and monitored more closely.

For all cancer sites combined, we observed a largely constant rate ratio (1.3) for the insulin-treated vs the nondiabetic populations, with longer duration of insulin use, at least during the first 10 years.

A direct effect of insulin on cancer initiation or promotion cannot be ruled out. In isolation, such an effect would be expected to start at a rate ratio of 1 and increase continuously by duration. We observed an initial rate ratio in insulin-treated vs non-insulin-treated diabetic patients of about 2.0, which fell to a level of 1.2 after 2 years among women, and slightly lower among men. This suggests that causes other than a direct insulin effect play a dominant role 


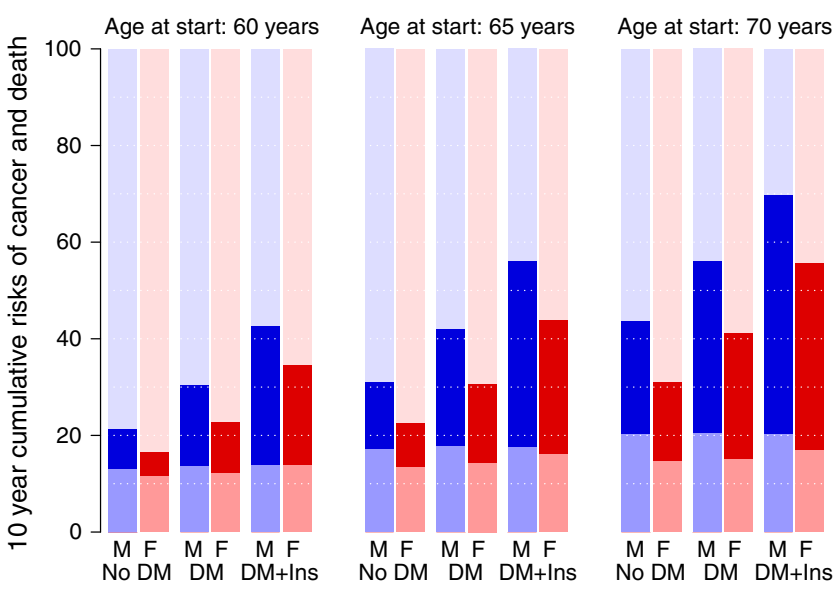

Fig. 6 Ten year cumulative risk for any type of cancer (light colour, bottom part) and death (full colour, middle part) and survival (pale colour, top part) from ages 60,65 and 70 years, starting in 1998. A non-diabetic individual is assumed to be immune from diabetes and a patient not using insulin is assumed to remain free from insulin. $\mathrm{M}$, male, F, female

just after treatment initiation, but a small, slowly increasing, effect of insulin use cannot be ruled out.

In the assessment of potential insulin effects, the 'all cancers' category is not really biologically meaningful. In this study, prostate, melanoma and colorectal cancer followed the pattern of decreasing rate ratio with longer insulin duration described above, whereas cancers of the stomach, lung, liver and kidney showed an indication of elevated cancer risk among insulin users.

Most strikingly, the rate ratio for pancreatic cancer showed a continued very sharp decline with increasing insulin duration. This effect is very likely due to reverse causation, where a pre-existing sub-clinical pancreatic cancer is the cause of diabetes, and first diagnosed after diabetes diagnosis. We found no indication that breast cancer incidence among diabetic patients differed from the non-diabetic population, regardless of duration of diabetes or insulin use. This is in line with the findings of Johnson et al. [22], and studies not taking duration into account [1-3], whereas Coughlin et al. [23] found an elevated risk of breast cancer in a mortality study.

\section{Relation to other studies}

Johnson et al. [22] recently analysed the effect of diabetes duration on cancer incidence in British Columbia, Canada, and found broadly similar results to ours, with elevated cancer incidence rate ratios in the first months after diagnosis, particularly for colorectal, prostate, lung and pancreatic cancers. They found a long-term elevated rate ratio only for cancer of the corpus uteri (endometrium) (rate ratio 1.6), and a reduced rate ratio of 0.8 for prostate cancer, consistent with our long-term estimates. Similarly, they also found that breast cancer rates were not affected by diabetes status or duration.

Our study also uniquely included information on insulin usage, as well as duration of diabetes and insulin treatment, and hence represents an essential addition to four recently published studies aiming at studying the effects of specific insulin analogues [24-27]. These four studies only performed internal comparisons between selected groups of diabetic patients and did not relate the cancer occurrence to that in the general population, because they focused on particular drug combinations. Moreover, these studies were largely confined to a short-term follow-up, that is, the time window where our study indicates that substantial confounding is present.

Our study specifically aimed at analysing duration effects, and hence only included persons diagnosed after 1995. Previous studies [1-3, 23] also included prevalent diabetes cases, and thus had an average length of diabetes duration and insulin duration that was longer than that in our study. Since the risk is highest for short duration of diabetes, we should expect to see higher rate ratios in our study's overall analysis (Fig. 1). For comparison, we did a simple analysis ignoring both diabetes duration and insulin treatment (see ESM [Fig. 2.13] for a comparison with previous major studies), and indeed found that this approach yielded rate ratios that were generally higher than in previous studies that ignored diabetes duration, but included prevalent cases of diabetes in the follow-up.

It could therefore be argued that analyses ignoring diabetes duration are meaningless, as they average over an influential variable (duration) with weights that are strongly dependent on the study design. Future studies of cancer occurrence among diabetic patients should, in our view, always include diabetes duration in order to distinguish between the markedly different risk periods we (and Johnson et al. [22]) detected.

The NDR does not distinguish between type 1 and type 2 diabetes, but a tabulation of cancers among diabetic patients diagnosed under age 35 years showed a total of 11 cases, so type 1 patients contribute minimally to the results.

\section{Cumulative risks}

Our study is the first to document that the significant differences in cancer incidence between diabetic patients with or without insulin therapy and non-diabetic individuals does not translate into major differences in the cumulative risk of cancer. Over a 10 year period, a diabetes diagnosis and initiation of insulin treatment were much stronger predictors of death from other causes than cancer. As a result of the limited 10 year survival probability for diabetes patients, the cumulative risk of a cancer diagnosis 
was similar to that of non-diabetic people. From a public health point of view, mortality from causes other than cancer seems to be a greater health issue among diabetic patients than the potential excess in cancer risk associated with diabetes and insulin therapy.

\section{Limitations}

Our study falls slightly short of recently published smaller studies, because the only available information on medication use was the date of second insulin purchase and no information on different insulin types or dosage was available. As is the case with all observational studies of effects of diagnosis and treatment, the current study is subject to confounding by indication, and the observed rate ratios between diabetes patients and insulin-treated patients relative to the general population cannot be interpreted causally.

\section{Strengths}

The study covered the entire Danish population, and thus there was no selection bias with regard to entry into the study population. Moreover, the study gives a precise picture of the actual incident cancer burden, as the DCR long history allowed for effective exclusion of all prevalent cancer cases at the start of our follow-up period. By including duration in our analyses, we were able to correct for the spurious effects seen in the first period after diagnosis of diabetes, and thus provide more credible estimates of the effects of diabetes and insulin use. Finally, the thorough Danish registration system ensured that all emigrations and deaths in the study population were properly accounted for.

\section{Conclusions}

Diabetic patients have elevated cancer incidence rates compared with the non-diabetic population, highest in the first year after diagnosis and the first year after initiation of insulin treatment. For non-users of insulin, the long-term rate ratio was 1.1 and for insulin users, the long-term rate ratio was 1.3 .

Diabetic patients not using insulin have higher long-term incidence rates of cancer of the colorectum, corpus uteri, kidney, liver and pancreas.

Diabetic patients using insulin have higher long-term incidence rates of cancer of the stomach, lung, kidney and liver.

Female diabetic patients have the same breast cancer incidence rates as those without diabetes, regardless of insulin therapy and duration.
Male diabetic patients have decreased rates of prostate cancer; the rate ratio decreases by duration of diabetes and, particularly, with the duration of insulin use.

Clinical effects of diabetes diagnosis and start of insulin treatment are clearly present, but long-term effects of diabetes per se, as well as insulin, cannot be ruled out.

Acknowledgements A preliminary version of this work, based only on 14 years of follow-up (not 15), and which contained an unfortunate computing error, was presented as a paper at the EASD meeting in September 2010 in Stockholm. In the abstract book (Abstract 147), a graph showed overall rate ratios of more than 1.5 associated with long-term use of insulin. The correct figure is 1.3 , as presented in Fig. 2 of this paper.

Duality of interest B. Carstensen and D.R. Witte are stockholders of NovoNordisk and employees at Steno Diabetes Center, a research hospital owned by NovoNordisk. S. Friis has no duality of interest in relation to this manuscript. No funding was received for this work, and no funding bodies played any role in the design, writing or decision to publish this manuscript.

Contribution statement The study was conceived collectively by the three authors, data preparation and statistical analysis was carried out by $\mathrm{BC}$, who also wrote the first draft of the paper. All three authors contributed equally to critical revision and interpretation of findings, and have all approved the final version of the paper.

\section{References}

1. Adami HO, McLaughlin J, Ekbom A et al (1991) Cancer risk in patients with diabetes mellitus. Cancer Causes Control 2:307-314

2. La Vecchia C, Negri E, Franceschi S, D'Avanzo B, Boyle P (1994) A case-control study of diabetes mellitus and cancer risk. Br J Cancer 70:950-953

3. Wideroff L, Gridley G, Mellemkjær L et al (1997) Cancer incidence in a population-based cohort of patients hospitalized with diabetes mellitus in Denmark. J Natl Cancer Inst 89:1360-1365

4. Bowker SL, Majumdar SR, Veugelers P, Johnson JA (2006) Increased cancer-related mortality for patients with type 2 diabetes who use sulfonylureas or insulin. Diabetes Care 29:254-258

5. Bonovas S, Filioussi K, Tsantes A (2004) Diabetes mellitus and risk of prostate cancer: a meta-analysis. Diabetologia 47:1071-1078

6. Calton BA, Chang SC, Wright ME et al (2007) History of diabetes mellitus and subsequent prostate cancer risk in the NIH-AARP Diet and Health Study. Cancer Causes Control 18:493-503

7. Pedersen CB (2011) The Danish civil registration system. Scand J Public Health 39(7 suppl):22-25

8. Carstensen B, Kristensen JK, Ottosen P, Borch-Johnsen K (2008) The Danish National Diabetes Register: trends in incidence, prevalence and mortality. Diabetologia 51:2187-2196

9. Carstensen B, Christensen JK, Marcussen MM, Borch-Johnsen K (2011) The National Diabetes Register. Scand J Public Health 39 (7 suppl):58-61

10. Storm HH, Michelsen EV, Clemmensen IH, Pihl J (1997) The Danish Cancer Registry-history, content, quality and use. Dan Med Bull 44:535-539

11. Gjerstorff ML (2011) The Danish Cancer Registry. Scand J Public Health 39(7 Suppl):42-45

12. Carstensen B (2007) Age-period-cohort models for the Lexis diagram. Stat Med 26:3018-3045 
13. Rosenbauer J, Strassburger K (2007) Comments on: "Ageperiod-cohort models for the Lexis diagram". Stat Med 27:1557-1561

14. Carstensen B (2007) Age-period-cohort models for the Lexis diagram (author's reply). Stat Med 27:1561-1564

15. Carstensen B (2011) \%Lexis: a SAS-macro for splitting follow-up time. http://192.38.117.59/ bxc/Lexis/Lexis.sas. Accessed 19 October 2011

16. Harrell FE (2001) Regression modeling strategies. Springer, New York

17. Putter H, Fiocco M, Geskus RB (2007) Tutorial in biostatistics: competing risks and multi-state models. Stat Med 26:2389-2430

18. Andersen PK, Abildstrøm SZ, Rosthøj S (2002) Competing risks as a multi-state model. Stat Methods Med Res 11:203-215

19. Cornfield J (1957) Estimation of the probability of developing a disease in the presence of competing risks. Am J Public Health Nations Health 47(5):601-607

20. R Development Core Team (2010) R: a language and environment for statistical computing. R Foundation for Statistical Computing, Vienna, Austria

21. Stocks T, Rapp K, Björge T et al (2009) Blood glucose and risk of incident and fatal cancer in the metabolic syndrome and cancer project (Me-Can): analysis of six prospective cohorts. PLoS Med 6:e1000201

22. Johnson JA, Bowker SL, Richardson K, Marra CA (2011) Timevarying incidence of cancer after the onset of type 2 diabetes: evidence of potential detection bias. Diabetologia 54:2263-2271

23. Coughlin SS, Calle EE, Teras LR, Petrelli J, Thun MJ (2004) Diabetes mellitus as a predictor of cancer mortality in a large cohort of US adults. Am J Epidemiol 159:1160-1167

24. Jonasson JM, Ljung R, Talbäck M, Haglund B, Gudbjörnsdottir S, Steineck G (2009) Insulin glargine use and short-term incidence of malignancies - a population-based follow-up study in Sweden. Diabetologia 52:1745-1754

25. Colhoun HM, SDRN Epidemiology Group (2009) Use of insulin glargine and cancer incidence in Scotland: a study from the Scottish Diabetes Research Network Epidemiology Group. Diabetologia 52:1755-1765, Erratum 52:2469

26. Currie CJ, Poole CD, Gale EA (2009) The influence of glucoselowering therapies on cancer risk in type 2 diabetes. Diabetologia 52:1766-1777

27. Hemkens LG, Grouven U, Bender R et al (2009) Risk of malignancies in patients with diabetes treated with human insulin or insulin analogues: a cohort study. Diabetologia 52:1732-1744 